\title{
¿Por qué duermen mal las mujeres embarazadas comparadas con las no gestantes? Estudio de corte transversal
}

¿Why pregnant women sleep poorly compared to non-pregnant women? Cross-sectional study

\author{
Stella M.Valiensi', Gustavo H. Izbizky²
}

\begin{abstract}
Resumen
Objetivos: Evaluar causas/motivos que fragmentan el sueño relacionados con los cambios fisiológicos, en los diferentes trimestres de embarazo y compararlos con los de las mujeres no gestantes. Identificar algunos trastornos del sueño en las mujeres embarazadas (ME) y compararlos con los de las mujeres no embarazadas (MNE). Método: Se realizó un estudio transversal utilizándose un cuestionario específico para recopilar causas que fragmentan el sueño, también se utilizó el Cuestionario del sueño de Oviedo, la Escala de somnolencia de Epworth y otros relacionados. Se invitó a participar a ME y MNE. Los resultados se expresaron en medidas de frecuencia, porcentaje, desviaciones medias y estándar (DE). Las diferencias se consideraron significativas si $p<0.05$ para todas las pruebas. Resultados: ME: I er Trimestre (T): $\mathrm{n}=$ 106, 2do.T: $\mathrm{n}=$ 104, 3er T: $\mathrm{n}=1$ I 0. Grupo control: $\mathrm{n}=$ 304 MNE. Al comparar ambos grupos encontramos: ME/ MNE: Ronquidos: $p=0.00$ I. Apnea: $p=0.89$. En MNE predominó: Síndrome de piernas inquietas: $p=0.01$. Bruxismo: $p=0.00$. Conclusión: Las ME reportaron sueño más fragmentado por casi todas causas fisiológicas, relacionadas con el embarazo, interrogadas, pero los únicos trastornos de sueño encontrados, por encuesta, fueron mayoritariamente los disturbios respiratorios, pesadillas y movimientos de las piernas. En MNE, sin embargo, encontramos más trastornos del sueño como Insomnio, hipersomnia y síndrome de piernas inquietas, bruxismo.
\end{abstract}

Palabras claves: Insomnio - Bruxismo - Disturbios respiratorios - Pesadillas - Embarazo.

\section{Abstract}

To evaluate causes/reasons that fragment sleep related to physiological changes in the different trimesters of pregnancy and compare them with those of non-pregnant women. To identify some sleep disorders in pregnant women (PW) and to compare them with those in non-pregnant women (NPW). Method: A cross sectional study was performed.A specific questionnaire was designed for the data collection of the study, Oviedo Sleep Questionnaire, Epworth Sleepiness Scale and others were also used. All the full term expectant mothers who attended the Obstetrics Department were invited to participate; non-pregnant students from College and teaching staff were used as control group.The results were expressed in measures of frequency, percent, mean and standard deviations (SD). Differences were considered significant if $p<0.05$ for all tests. Results: PW: Ist Trimester (T): $n=106,2 n d . T: n=104,3 r d T: n=1$ I0. Control group: $n=304$ NPW. When comparing both groups we find: ME / MNE: Snoring: $p=0.001$. Apnea: $p=0.89$. In NPW, the following predominated: Restless legs syndrome: $p=0.01$. Bruxism: $p=0.00$. Conclusions: The $P W$ reported more fragmented sleep due to almost all physiological causes, related to pregnancy, who were questioned, but the only sleep disorders found, by survey, were mainly respiratory disorders, nightmares and leg movements. In NPW, however, we found more sleep disorders such as insomnia, hypersomnia and restless legs syndrome, bruxism.

Keywords: Pregnancy - Insomnia - Bruxism - Restless legs syndrome - Snoring - Apnea.

\footnotetext{
1.Médica Neuróloga. Sección de Medicina del sueño. Servicio de Neurología del Hospital Italiano de Buenos Aires.

2.Médico Obstetra. Subjefe del Servicio de Obstetricia del Hospital Italiano de Buenos Aires. Instituto Universitario Hospital Italiano de Buenos Aires.
} 


\section{Introducción}

El patrón de sueño sufre cambios importantes relacionados con alteraciones anatómicas, fisiológicas y conductuales durante la gestación. Se recomendado enfáticamente que todo el equipo de salud, conozca las características normales y los trastornos del sueño durante el embarazo para ofrecer una serie de pautas o recomendaciones que contribuyan a mejorar la salud física y mental presente y futura, de la gestante y del feto (The American College of Obstetricians and Gynecologists Committee, 2015).

Entre 66 y 94\% de las mujeres embarazadas (ME) reconocen sufrir algún problema relacionado con el sueño como somnolencia, dificultad para la consolidación del sueño o sensación de sueño poco reparador (Santiago et al., 2001). Según el momento de la gestación se describen, por ejemplo, en el primer trimestre (1er.T.) (Manber \& Armitage, 1999) fatiga, somnolencia diurna, despertares por náuseas, aumento de la frecuencia urinaria, molestias físicas como dolor de espalda y cambios de humor (Mindell \& Jacobson, 2000; Okun et al., 2011; Lee \& Zaffke, 1999; Neau et al., 2009) y otros cambios denominados "fisiológicos" asociados a los cambios hormonales específicos de cada trimestre.

Durante el segundo trimestre (2do.T), los ronquidos, la acidez, las contracciones uterinas irregulares, los calambres, el síndrome de piernas inquietas (SPI) (Guilleminault et al., 2000), los sueños vívidos y el dolor del cuello, de la espalda, y de las articulaciones, suelen causar interrupción del sueño. En el tercer trimestre (3er. T.), los cambios físicos producidos por el rápido crecimiento del útero y los cambios que producen las fluctuaciones hormonales, son las principales causas que fragmentan el sueño (Tsai et al., 2012). Las mujeres también atribuyen su pérdida de sueño a factores internos y externos como las necesidades de sus otros hijos o el medio ambiente (Hall et al., 2009).

Los trastornos o disturbios del sueño, no solo se manifiestan mientras el individuo está dormido sino que se prolongan durante el día, es decir, tienen repercusión a nivel médico con morbilidad y mortalidad asociadas, tanto orgánica (p. ej., cardiovascular) como psicológica, a nivel de las relaciones interpersonales y el rendimiento académico y laboral. La Clasificación Internacional de desórdenes del Sueño, en su tercera versión (ICSD 3-2014), señala, como consecuencia de los trastornos del sueño, alteraciones tanto en el plano fisiopatológico, como en el social y en el epidemiológico. Se calcula que al menos un $10 \%$ de la población general sufre algún trastorno del sueño clínicamente significativo, con repercusión social e intrapersonal.
En las ME, al igual que en la población general, también se pueden presentar disturbios del sueño que repercuten en la calidad de vida y varían según la ICSD 3-2014, desde el insomnio, a la hipersomnia, los trastornos del movimiento, los trastornos respiratorios (ronquidos y apneas del sueño) y las parasomnias (Dzaja et al., 2005; Alvarez-Aguilar et al., 2010), así como trastornos neurológicos y médicos relacionados con el sueño, y desórdenes del sueño inducidos por sustancias.

Los síntomas de insomnio (problemas para conciliar el sueño, despertares nocturnos, despertarse temprano por la mañana, con consecuencias durante el día como fatiga, irritabilidad y pérdida de concentración) suelen ser las manifestaciones de disturbios del sueño más frecuentes (LeeGay, 2004; Watson et al., 2006; Meijer \&Van den Wittenboer, 2007). La hipersomnia es más prevalente durante el 1er. y 3er. T. del embarazo (Lopes, 2004).

Los trastornos del movimiento principalmente el síndrome de piernas inquietas (SPI), se afecta a un 11 a un $27 \%$ de las ME y se produce fundamentalmente durante el 3er. T., remitiendo la sintomatología luego del parto (García et al., 2006; Lyn, 2007; Tunc et al., 2007; Ferini-Strambi \& Granieri, 2004; Lamberg, 2006).

Entre los trastornos respiratorios, el ronquido tiene una prevalencia del 45\% en las ME (Calaora-Tournadre et al., 2006; Köken et al., 2007), mientras que la apnea del sueño tiene una prevalencia similar a la de la población general (Facco et al., 2017; Domingo et al., 2006; Langner et al., 2007; Roush \& Bell, 2003; Pérez-Chada, 2007).

La literatura internacional aporta múltiples datos respecto de estos problemas del sueño en ME pero en nuestro país existen pocos trabajos que hayan investigado las causas del mal dormir de las mujeres durante el embarazo (Pérez-Chada, 2007). El presente estudio fue diseñado para indagar sobre ese fenómeno, con los siguientes objetivos: 1) evaluar en forma subjetiva las causas que fragmentan el sueño en ME y compararlas con MNE y, 2) evaluar en forma subjetiva algunos trastornos del sueño en los diversos trimestres en las ME y compararlos con MNE.

\section{Pacientes y métodos}

Se realizó un estudio de corte transversal. Se incluyeron $320 \mathrm{ME}$ que consultaron en el Servicio de Obstetricia de un Centro Universitario polivalente de alta complejidad, durante los diferentes trimestres, participando cada mujer, sólo una vez. Como grupo control se seleccionaron $301 \mathrm{MNE}$ sanas, todas ellas estudiantes, docentes universitarias o personal femenino de la institución. 
Los criterios de inclusión fueron: ME y MNE que aceptaron participar, edades comprendidas entre 18 a 45 años y nivel de educación de 12 o más años. Se incluyeron $320 \mathrm{ME}$ de las cuales, 106 mujeres cursaban $\leq 13$ semanas de gestación (1er. Trimestre); 104 cursaban las semanas 14-27 de gestación (2do. Trimestre) y 110 cursaban $\geq 28$ semanas de gestación (3er Trimestre). Como controles, se incluyeron en el estudio $304 \mathrm{MNE}$.

Previo consentimiento, se entregó a las participantes una encuesta anónima, de auto-reporte.

La encuesta incluía antecedentes sociodemográficos y clínicos: edad, estado civil, ocupación, embarazos previas, hábitos, antecedentes de disfunción tiroidea, hiperlipidemia e hipertensión. Se les preguntaba mediante un cuestionario específico, sobre posibles causas que fraccionaran su sueño en los últimos tres meses. Se les preguntaba las posibles causas que fragmentaran su sueño, conforme a causas fisiológicas citadas, producidas ya sea por cambios hormonales, evolución del embarazo o aumento de peso, entre otras causas.

Para analizar las otras variables relacionadas con trastornos del sueño se aplicaron cuestionarios utilizados en población general para abordar los diversos trastornos del sueño. En esta oportunidad, se utilizó el Cuestionario Oviedo de Sueño (COS) (García-Portilla et al., 2009), que consiste en una entrevista semi-estructurada de ayuda diagnóstica para insomnio. Dicha herramienta también valora la existencia de hipersomnia (excesiva somnolencia diurna) y, mediante otras preguntas, permite valorar otros disturbios del sueño como parasomnias (específicamente la presencia de pesadillas), movimientos de piernas durante el sueño, presencia de disturbios respiratorios como ronquidos y apneas. El COS consta de un total de 15 ítems donde describe distintos aspectos de su conducta durante el último mes, pero nosotros preguntamos los últimos 3 meses. Con el análisis de este cuestionario, se obtiene:

1. una valoración subjetiva de la satisfacción con el sueño;

2. una valoración cualitativa de presencia de insomnio o hipersomnia y

3. una valoración cuantitativa del insomnio en el caso de que éste sea el trastorno de sueño, presente (Bobes et al., 2000). La presencia de pesadillas, movimientos periódicos de piernas, ronquidos, apneas, se consideró como presente si se presentaba, más de 3 veces por semana, en el último trimestre.
Para evaluar somnolencia diurna, se utilizó la Escala de Somnolencia de Epworth (ESS) (Johns, 1991), considerándose somnolencia diurna excesiva a una puntuación superior a 10 (Leigh Signal et al., 2014).

El diagnóstico de SPI, se estableció mediante preguntas que comprendían los cuatro elementos esenciales para definirlo (Allen et al., 2003; Hening et al., 2004). Las pacientes se clasificaron como que presentaban el SPI, si respondían "sí" a todas las preguntas.

\section{Análisis estadístico}

La información obtenida fue transcrita a una base de datos informatizada, utilizando el programa SPSS 25.0 para Windows, que permitió el análisis descriptivo de los datos utilizando medidas de frecuencia, media, moda, mediana y desviación estándar.

Las variables categóricas se presentaron en número absolutos y porcentajes.

Se utilizó el t-test de acuerdo con la distribución de datos continuos y se realizó análi-sis de varianza entre grupos con corrección Bonferroni. Las proporciones se analizaron con la prueba $\chi 2$. En todas las pruebas estadísticas se tomó como valor de referencia de la significación estadística $\mathrm{p}<0.05$.

\section{Consideraciones éticas}

Todos los cuestionarios fueron anónimos por lo que no se requirió consentimiento escrito. Todos los procedimientos realizados siguieron las reglas de la Declaración de Helsinki de 2013 y su carta de enmienda, para investigación en humanos. El estudio fue aprobado por el Comité de Ética de Protocolos de Investigación con el número 2032.

\section{Resultados}

De 700 encuestas distribuidas, se obtuvieron para el análisis 304 de MNE y 320 encuestas de ME. Del 1r. T. $=106$, del 2 do T. $=104$ y del 3 er T. $=110$ encuestas. Las características sociodemográficas de la población de SE ME detallan en la tabla 1.

Al comparar ambas poblaciones, encontramos que las MNE consumían más café y mate, fumaban más y consumían más drogas que las ME (ver tabla 2).

En la tabla 3, se compararon las causas interrogadas que fragmentaban el sueño en las ME según los diversos trimestres, y en la tabla 4 se compararon los mismos motivos, entre ME y MNE, donde dolor, calambres, pensamientos negativos, urgencia miccional, no poder rotar en la cama, vómitos, reflujo ácido, dificultad para respirar, ronquidos, por razones/causas 
Tabla I. Características sociodemográficas en los diferentes trimestres del embarazo

\begin{tabular}{|c|c|c|c|c|c|c|c|c|}
\hline \multirow[t]{2}{*}{ Variables generales } & \multicolumn{2}{|c|}{ Datos globales } & \multicolumn{2}{|c|}{$\leq 13$ semanas } & \multicolumn{2}{|c|}{ 14-27 semanas } & \multicolumn{2}{|c|}{$\geq \mathbf{2 8}$ semanas } \\
\hline & $\mathrm{n}$ & $\%$ & $\mathrm{n}$ & $\%$ & $\mathrm{n}$ & $\%$ & $\mathrm{n}$ & $\%$ \\
\hline \multicolumn{9}{|l|}{ Edad (años) } \\
\hline$<19$ & 6 & 1.9 & 2 & 1.9 & 2 & 1.9 & 2 & 1.8 \\
\hline $20-29$ & 99 & 31.0 & 31 & 29.2 & 37 & 35.6 & 31 & 28.2 \\
\hline $30-39$ & 189 & 59.0 & 61 & 57.5 & 61 & 58.6 & 67 & 61.0 \\
\hline$\geq 40$ & 26 & 8.1 & 12 & 11.3 & 4 & 3.9 & 10 & 9.0 \\
\hline \multicolumn{9}{|l|}{ Índice de masa corporal } \\
\hline$<$ 18.5. Delgadez & 14 & 4.4 & 3 & 2.8 & 6 & 5.8 & 5 & 4.5 \\
\hline I8.5-24. Peso normal & 137 & 42.8 & 62 & 58.5 & 47 & 45.2 & 28 & 25.5 \\
\hline 25-29. Sobrepeso & 117 & 36.5 & 27 & 25.5 & 35 & 33.6 & 55 & 50.0 \\
\hline 30-34.9. Obesidad tipo I & 37 & 11.6 & 12 & 11.3 & 11 & 10.6 & 14 & 12.7 \\
\hline 35-39. Obesidad tipo II & 12 & 3.8 & 2 & 1.9 & 4 & 3.8 & 6 & 5.4 \\
\hline$\geq 40$. Obesidad tipo III & 3 & 0.9 & 0 & 0 & 1 & 1.0 & 2 & 1.9 \\
\hline \multicolumn{9}{|c|}{ Número de embarazos e hijos previos } \\
\hline 0 & 193 & 60.3 & 75 & 70.8 & 58 & 55.8 & 60 & 54.5 \\
\hline I & 92 & 28.7 & 16 & 15.0 & 35 & 33.7 & 41 & 37.3 \\
\hline 2 & 28 & 8.7 & 11 & 10.4 & 9 & 8.6 & 8 & 7.3 \\
\hline$\geq 3$ & 7 & 2.3 & 4 & 3.8 & 2 & 1.9 & 1 & 0.9 \\
\hline
\end{tabular}

relacionadas a esposo o hijos, fueron causas estadísticamente significativas que fragmentaban el sueño en las ME.

En la tabla 5, se detalla trastornos del sueño en diversos trimestres del embarazo, obtenidos por datos recolectados a través de COS, como que en el 3er. T., las ME, están más insatisfechas con su sueño, tienen más insomnio y más pesadillas. En el 1er T. tienen más hipersomnia. No hubo diferencias significativas en el puntaje obtenido mediante la ESS, cuestionario para SPI, disturbios respiratorios, pesadillas o movimientos periódicos de piernas.

En la tabla 6, se comparan los trastornos del sueño entre ME y MNE. Se observa que los disturbios respiratorios durante el sueño, movimientos de piernas y pesadillas que fueron más prevalentes en las ME pero insomnio, hipersomnia y SPI obtuvimos fue significativo, a favor de las MNE.

\section{Discusión}

Los motivos "fisiológicos" relacionados con la evolución del embarazo, que fraccionan y/o fragmentan el sueño nocturno en las $\mathrm{ME}$, con diferencia significativas con respecto a MNE, fueron: 1) la necesidad de orinar provocada por el aumento progresivo del tamaño del útero, la compresión de la vejiga resultante, el aumento del flujo sanguíneo renal y la relajación de la musculatura lisa de la pelvis renal, 2) la limitación de la rotación del cuerpo en la cama, una posición incómoda para dormir o molestias musculares, generadas por el aumento de peso, c) los dolores dorsales producidos por cambios músculo-esqueléticos, retención de fluidos en el tejido conectivo y exageración de la lordosis lumbar fisiológica, d) los calambres; éstos fueron menos más prevalentes en nuestras ME que los reportados en la literatura $(21 \%, 57 \%, 75 \%$ en los sucesivos trimestres de la gestación), aunque se mantuvo la diferencia entre el 1er y 3er T (Baratte-Beebe \& Lee, 1999).

Las náuseas y los vómitos, causados por efectos inhibitorios de la progesterona sobre el músculo liso gastrointestinal, el aumento de la presión gástrica y el desplazamiento del esfínter esofágico inferior por el útero grávido, justifican el reflujo gastroesofágico (contenido gástrico que asciende al esófago) que afectó a la mayoría de las ME (Ali \& Egan, 2007; Rey et al., 2007; Morey et al., 1997; Malfertheiner et al., 2012).

El sonambulismo, la somniloquia y el bruxismo durante el sueño suelen disminuir durante el embarazo especialmente en las nulíparas (Facco et al., 2010). La somniloquia fue mayor en las ME en el 3er T., pero no hubo diferencia con MNE. Con respecto a las pesadillas, algunos autores afirman que disminuyen (Hed- 
Tabla 2. Comparación de características sociodemográficas, hábitos y comorbilidades entre ME y MNE

\begin{tabular}{|c|c|c|c|c|c|}
\hline & \multicolumn{2}{|c|}{$M E n=320$} & \multicolumn{2}{|c|}{ MNE $n=304$} & $\mathrm{P}$ \\
\hline Edad (años, media \pm DS) & \multicolumn{2}{|c|}{$31.6 \pm 5.3$} & \multicolumn{2}{|c|}{$30.8 \pm 7.3$} & 0.09 \\
\hline IMC (índice de masa corporal) & \multicolumn{2}{|c|}{$25.9 \pm 5.3$} & \multicolumn{2}{|c|}{$22.7 \pm 3.8$} & $<0.001$ \\
\hline Número de hijos & $\mathrm{n}$ & $\%$ & $\mathrm{n}$ & $\%$ & \\
\hline 0 & 193 & 60 & 227 & 75 & \\
\hline I & 92 & 29 & 42 & 14 & \\
\hline 2 & 28 & 9 & 28 & 9 & \\
\hline 3 o más & 7 & 2 & 5 & 2 & 0.001 \\
\hline \multicolumn{6}{|l|}{ Empleo } \\
\hline Desempleada & 68 & 21 & 74 & 24.6 & \\
\hline Trabaja por la mañana & 249 & 77 & 224 & 75 & \\
\hline Trabaja por la tarde & 3 & 1 & I & 0.4 & 0.395 \\
\hline \multicolumn{6}{|l|}{ Actividad física } \\
\hline Actividad física, $\geq 1$ hora por semana & 75 & 24 & 164 & 54 & 0.001 \\
\hline \multicolumn{6}{|l|}{ Comorbilidades } \\
\hline Hipertensión & 12 & 4 & 8 & 3 & 0.595 \\
\hline Disfunción tiroidea & 48 & 15 & 52 & 17 & 0.409 \\
\hline Dislipidemia & 18 & 6 & 24 & 8 & 0.208 \\
\hline \multicolumn{6}{|l|}{ Bebidas/infusiones } \\
\hline Consume café & 142 & 44.4 & 207 & 68 & $<0.001$ \\
\hline Consume té & 149 & 46.6 & 155 & 51 & 0.27 \\
\hline Consume mate llex Paraguriensis & 163 & 51 & 222 & 73 & $<0.001$ \\
\hline \multicolumn{6}{|l|}{ Tabaco /drogas } \\
\hline Fuma $\geq$ I cigarrillo por día & 13 & 4 & 52 & 17 & $<0.001$ \\
\hline Consume marihuana/otros & 4 & 1.25 & 36 & 11.7 & $<0.001$ \\
\hline Consume alcohol & 7 & 2.2 & 100 & 33 & $<0.001$ \\
\hline
\end{tabular}

Para el análisis del valor de $\mathrm{P}$ se usó $\mathrm{X} 2$ test.

man et al., 2002) y otros que aumentan (Mindell \& Jacobson, 2000) conforme progresa la edad gestacional con valores de hasta $25 \%$. En este estudio se encontró una diferencia significativa como causa que fragmenta el sueño entre el ler y $3 \mathrm{er} \mathrm{T}$. Al comparar mediante COS entre mujeres, la diferencia fue significativa y reportada en el $43.7 \%$ de ME. Probablemente por tener un sueño fragmentado, recuerdan más las pesadillas en su proceso de maduración psicológica sobre la situación futura (Facco et al., 2010).

La queja de bruxismo como motivo de fragmentación del sueño fue el doble en las MNE. En las ME, el bruxismo que se manifestaba en el $10.3 \%$ en el $1 \mathrm{er} \mathrm{T}$. fue decreciendo a medida que avanzaba la gestación. Algunos autores han referido que el bruxismo se atenúa o desaparece durante el embarazo.
Otro de los trastornos del sueño, fueron los disturbios respiratorios que fueron significativos en las MNE (Pien et al., 2005). Es primordial indagar sobre la presencia de estos eventos en las ME dado su relación con la aparición de hipertensión gravídica, preeclampsia y bajo peso del recién nacido (Langner et al., 2007; Roush \& Bell, 2003).

La prevalencia en la población general en Argentina del SPI es de 20.2\% (Persi, 2009). La prevalencia reportada a nivel internacional en ME es de 11 a $27 \%$. En el presente estudio se constataron valores similares, que se incrementaron en el 3er.T, aunque de forma estadísticamente no significativa. Llamativamente, fue mayor el porcentaje de este trastorno del sueño, en las MNE (Ellis et al., 2012; Fernandez-Alonso et al., 2012; Kizilirmak et al., 2012; Dorheim et al., 2014). 
Tabla 3. Comparación de diversos motivos que fragmentan el sueño en los diversos trimestres del embarazo

\begin{tabular}{|c|c|c|c|c|}
\hline \multirow[t]{3}{*}{ Motivos que fragmentan el sueño } & \multirow{3}{*}{$\begin{array}{c}\leq 13 \text { semanas } \\
n=106 \\
\%\end{array}$} & \multirow{3}{*}{$\begin{array}{c}4-2 \text { semanas } \\
n=104 \\
\%\end{array}$} & \multirow{2}{*}{$\begin{array}{c}\geq 28 \text { semanas } \\
n=110\end{array}$} & \multirow[b]{3}{*}{$\mathrm{P}$} \\
\hline & & & & \\
\hline & & & $\%$ & \\
\hline Cefalea & 8.4 & 18.2 & 14.5 & 0.20 \\
\hline Pensamientos agradables & 5.6 & 11.5 & 11.8 & 0.12 \\
\hline Pensamientos desagradables/feos & 29.2 & 37.5 & 43.6 & 0.02 \\
\hline Pesadillas & 17.9 & 36.5 & 36.9 & 0.03 \\
\hline Sensación de agitación & 3.7 & 7.6 & 8.1 & 0.19 \\
\hline Dolor en región del cuello & 18.8 & 24 & 23.6 & 0.40 \\
\hline Dolor lumbar & 17.9 & 32.6 & 50 & 0.001 \\
\hline Dolor articular & 3.3 & 6.7 & 24.5 & 0.001 \\
\hline Dolor de piernas & 13.2 & 16.3 & 39.0 & 0.001 \\
\hline Intensa necesidad de mover las piernas & 7.5 & 16.3 & 16.3 & 0.06 \\
\hline Movimientos de piernas & 7.5 & 14.0 & 15.4 & 0.08 \\
\hline Calambres de miembros inferiores & 13.2 & 21.1 & 50 & 0.001 \\
\hline Frío o sensación de calor & 44.3 & 35.5 & 42.7 & 0.82 \\
\hline Necesidad de orinar & 78.3 & 82.6 & 95.4 & 0.001 \\
\hline Sudoración & 2.8 & 4.8 & 27 & 0.95 \\
\hline Dormir en posición incómoda & 30.1 & 45.1 & 57.2 & 0.001 \\
\hline No poder rotar en la cama & 11.3 & 28.8 & 67.2 & 0.001 \\
\hline Necesidad de comer a la noche & 5.6 & 10.5 & 17.2 & 0.007 \\
\hline Reflujo gastroesofágico & 7.5 & 12.5 & 15.4 & 0.007 \\
\hline Disconfort abdominal & 13.2 & 13.4 & 10.0 & 0.07 \\
\hline Eructos & 6.6 & 2.8 & 4.5 & 0.48 \\
\hline Nausea & 16 & 11.5 & 5.4 & 0.01 \\
\hline Vómitos & 6.6 & 11.5 & 7.2 & 0.87 \\
\hline Palpitaciones & 4.7 & 5.7 & 5.4 & 0.81 \\
\hline Disnea, falta de aire & 7.5 & 10.5 & 11.8 & 0.29 \\
\hline Ronquidos & 8.4 & 2.8 & 7.2 & 0.72 \\
\hline Pausas respiratoria & 0 & 1.9 & 3.6 & 0.04 \\
\hline Somniloquia (hablar dormida) & 2.8 & 3.8 & 4.5 & 0.50 \\
\hline Sonambulismo & 0.9 & 1.9 & 1.8 & 0.60 \\
\hline Debido a los hijos o pareja & 23.5 & 32.6 & 33.6 & 0.10 \\
\hline Bruxismo & 10.3 & 9.6 & 9.0 & 0.75 \\
\hline
\end{tabular}

Diferencia entre el ler y 3er T del embarazo. + diferencia entre 2do y 3er T de embarazo.

Para el análisis del valor de $\mathrm{P}$ se usó ${ }^{€}$ test $\mathrm{X} 2$.

Como ambas poblaciones estudiadas tienen perfiles genéticos similares, cabe suponer que otros factores tales como características de la alimentación, consumo de sustancias estimulantes como mate Ilex Paraguriensis, o exceso de cafeína (que fueron superiores significativamente entre las MNE), pudieron influir en el resultado, pero deberían realizarse más estudios para corroborar esta hipótesis, comparando ambas poblaciones.

Según algunas encuestas el 52\% de las ME se quejan de insomnio (National Sleep Foundation, 2007), y en el 3er T la queja se incrementa (Vgontzas et al., 2001). En este estudio se utilizó el cuestionario COS para valorar insomnio y se encontró que las ME se 
Tabla 4. Comparación de diversos motivos que fragmentan el sueño entre mujeres embarazadas (ME) y mujeres no embarazadas (MNE)

\begin{tabular}{|c|c|c|c|c|c|}
\hline \multirow[t]{3}{*}{ Motivos que fragmentan el sueño } & \multirow{2}{*}{\multicolumn{2}{|c|}{$\begin{array}{c}\text { ME } \\
n=320\end{array}$}} & \multirow{2}{*}{\multicolumn{2}{|c|}{$\begin{array}{c}\text { MNE } \\
n=304\end{array}$}} & \multirow[b]{3}{*}{$\mathrm{P}$} \\
\hline & & & & & \\
\hline & $\mathrm{n}$ & $\%$ & $\mathrm{n}$ & $\%$ & \\
\hline Cefalea & 44 & 13.8 & 27 & 11.0 & 0.315 \\
\hline Pensamientos agradables & 31 & 9.7 & II & 4.5 & 0.018 \\
\hline Pensamientos desagradables/feos & 118 & 36.8 & 95 & 38.5 & 0.699 \\
\hline Pesadillas & 91 & 28.4 & 56 & 22.7 & 0.120 \\
\hline Sensación de agitación & 21 & 6.6 & 14 & 5.7 & 0.661 \\
\hline Dolor en región del cuello & 71 & 22.2 & 49 & 19.8 & 0.49 \\
\hline Dolor lumbar & 108 & 33.8 & 47 & 19 & $<0.001$ \\
\hline Dolor articular & 38 & 11.9 & 15 & 6.0 & 0.019 \\
\hline Dolor de piernas & 74 & 23.1 & 20 & 8.0 & $<0.001$ \\
\hline Intensa necesidad de mover las piernas & 43 & 13.4 & 10 & 4.0 & $<0.001$ \\
\hline Movimientos de piernas & 40 & 12.5 & 23 & 11.11 & 0.231 \\
\hline Calambres de miembros inferiores & 91 & 28.44 & 33 & 13.36 & 0.000 \\
\hline Frío o sensación de calor & 131 & 40.94 & 86 & 34.82 & 0.137 \\
\hline Necesidad de orinar & 274 & 85.62 & 121 & 48.99 & 0.000 \\
\hline Sudoración & 11 & 3.44 & 3 & 1.21 & 0.091 \\
\hline Dormir en posición incómoda & 142 & 44.38 & 83 & 33.60 & 0.009 \\
\hline No poder rotar en la cama & 116 & 36.25 & 16 & 6.48 & 0.000 \\
\hline Necesidad de comer a la noche & 36 & 11.25 & 14 & 5.67 & 0.020 \\
\hline Dolor de estómago & 38 & 11.88 & 10 & 4.07 & $<0.001$ \\
\hline Disconfort abdominal & 39 & 12.19 & 15 & 6.07 & 0.014 \\
\hline Eructos & 15 & 4.69 & 5 & 2.02 & 0.088 \\
\hline Nausea & 35 & 11 & 5 & 2 & $<0.001$ \\
\hline Vómitos & 27 & 8.44 & 3 & 1.21 & 0.000 \\
\hline Reflujo gástrico & 79 & 24.69 & 8 & 3.24 & 0.000 \\
\hline Palpitaciones & 17 & 5.31 & 9 & 3.64 & 0.346 \\
\hline Disnea, falta de aire & 32 & 10 & 3 & 1.21 & 0.000 \\
\hline Ronquidos & 20 & 6.25 & 6 & 2.43 & 0.031 \\
\hline Pausas respiratoria & 6 & 1.88 & 5 & 2.02 & 0.898 \\
\hline Somniloquia (hablar dormida) & 12 & 3.75 & 15 & 6.10 & 0.194 \\
\hline Sonambulismo & 5 & 1.56 & 3 & 1.21 & 0.728 \\
\hline Debido a los hijos o pareja & 96 & 30 & 39 & 15.79 & 0.000 \\
\hline Bruxismo & 31 & 9.69 & 61 & 24.7 & 0.000 \\
\hline
\end{tabular}

quejan más de insomnio y tienen menor satisfacción con el sueño en el 3er T., con respecto al 1er T.; pero el porcentaje de insomnio global (por este método) fue solo de $17.6 \%$, es decir, significativamente superior comparado con el obtenido por las MNE. Sin embargo, las MNE reportan tener más sueño reparador que las $\mathrm{ME}$, con una diferencia significativa. Postulamos que el mal descanso de las ME se explica mejor por la existencia de un sueño fragmentado por causas fisiológicas y/o ambientales, más que por trastornos del sueño en sí mismo.

Se han adjudicado razones como el hiper arousal, el incremento de secreción de ACTH y cortisol, cambios en el GABA (Manber \& Armitage, 1999; Lancel et 
Tabla 5. Comparación de diversos trastornos del sueño según diversos cuestionarios y preguntas relacionadas, en los distintos trimestres de embarazo

\begin{tabular}{|c|c|c|c|c|c|c|c|}
\hline \multirow{3}{*}{$\begin{array}{l}\text { Disturbios del sueño según cuestionarios y preguntas } \\
\text { relacionadas }\end{array}$} & \multirow{2}{*}{\multicolumn{2}{|c|}{$\begin{array}{c}\leq 13 \text { semanas } \\
n=106\end{array}$}} & \multirow{2}{*}{\multicolumn{2}{|c|}{$\begin{array}{c}\text { 14-27 semanas } \\
n=104\end{array}$}} & \multirow{2}{*}{\multicolumn{2}{|c|}{$\begin{array}{c}\geq 28 \text { semanas } \\
n=110\end{array}$}} & \multirow{2}{*}{$\mathbf{p}$} \\
\hline & & & & & & & \\
\hline & $3.9 *$ & 1.7 & $3.9+$ & 1.7 & $3.3^{*}+$ & 1.6 & $* 0.017$ \\
\hline (Media $\pm \mathrm{DS})$ & & & & & & & +0.008 \\
\hline Insomnio global según COS (media $\pm \mathrm{DS})$ & $16.8^{8}$ & 4.4 & 17.4 & 4.9 & $18.7^{\&}$ & 5.4 & $* 0.028$ \\
\hline Hipersomnia según COS (Media \pm DS) & 5.4 & 2.2 & 5.3 & 1.9 & 5.2 & 1.6 & 0.02 \\
\hline Insomnio CIE I0 según COS (n/\%) & 17 & 16.4 & 18 & 17.3 & 18 & 16.3 & 0.968 \\
\hline Insomnio DSM-IV según COS (n/\%) & 4 & 3.77 & 2 & 1.9 & 5 & 4.5 & 0.559 \\
\hline Ronquidos según COS (n/\%) & 30 & 28.3 & 20 & 19.2 & 28 & 25.4 & $0.63^{€}$ \\
\hline Ronquidos con apneas según $\operatorname{COS}(n / \%)$ & 32 & 30.2 & 31 & 29.9 & 37 & 33.6 & $0.58^{€}$ \\
\hline Movimientos de piernas según COS (n/\%) & 31 & 29.2 & 28 & 26.9 & 33 & 30.0 & $0.89^{€}$ \\
\hline Pesadillas según COS (n/\%) & 48 & 45.3 & 46 & 44.2 & 46 & 41.8 & $0.60^{€}$ \\
\hline Puntaje de Escala de somnolencia de Epworth (Media \pm DS) & 6.5 & 4.8 & 6.9 & 4.9 & 5.7 & 4.3 & 0.435 \\
\hline Cuestionario de SPI (n/\%) & 19 & 17.9 & 26 & 25 & 28 & 25.4 & 0.34 \\
\hline
\end{tabular}

Análisis de varianza con corrección Bonferroni: *comparación entre ler y 3er T $p=0.019,+$ comparación entre 2 do y 3er T $p=0.008$; ${ }^{\text {comparación }}$ entre el ler y el 3er $\mathrm{T} p=0.019$.

* Diferencia entre el ler y 3er T del embarazo. + diferencia entre 2do y 3er T de embarazo. Para el análisis del valor de $\mathrm{P}$ se usó ${ }^{\epsilon}$ test $\mathrm{X} 2$.

Tabla 6. Comparación de diversos trastornos del sueño según cuestionarios y preguntas relacionadas, entre mujeres embarazadas (ME) y no embarazadas (MNE)

\begin{tabular}{|c|c|c|c|c|c|}
\hline \multirow{3}{*}{$\begin{array}{l}\text { Disturbios del sueño según cuestionarios y preguntas relacionadas } \\
\text { \#COS I Satisfacción con el sueño (media } \pm \text { DS) }\end{array}$} & \multirow{2}{*}{\multicolumn{2}{|c|}{$\begin{array}{c}\text { ME } \\
n=320\end{array}$}} & \multirow{2}{*}{\multicolumn{2}{|c|}{$\begin{array}{c}\text { MNE } \\
n=304\end{array}$}} & \multirow{3}{*}{$\begin{array}{c}P \\
0.57\end{array}$} \\
\hline & & & & & \\
\hline & 3.7 & 1.7 & 3.8 & 1.5 & \\
\hline Insomnio global según COS (media \pm DS) & 17.6 & 4.9 & 18.9 & 5.4 & 0.004 \\
\hline Hipersomnia según COS (n/\%) & 54 & 16.8 & 39 & 13 & 0.09 \\
\hline Insomnio CIE I0 según COS (n/\%) & 53 & 16.5 & 65 & 21.3 & 0.22 \\
\hline Insomnio DSM-IV según COS (n/\%) & 11 & 3.4 & 4 & 1.3 & 0.06 \\
\hline \# COS 2.1 Dificultades para iniciar el sueño & 22 & 6.8 & 27 & 8.9 & 0.35 \\
\hline \# COS 2.1 Sueño reparador & 29 & 9 & 48 & 15.8 & 0.01 \\
\hline \# COS 5. Despertar precoz ( $\geq$ I hora) & 36 & 11.2 & 32 & 10.5 & 0.77 \\
\hline \# COS I0.A. Ronquidos (n/\%) & 78 & 24.0 & 39 & 12.8 & 0.001 \\
\hline \# C OS I0.B. Ronquidos con pausas (n/\%) & 100 & 31.2 & 3 & 2.9 & 0.001 \\
\hline \# COS 10. C. Movimientos de piernas (n/\%) & 92 & 28.7 & 37 & 12.1 & 0.001 \\
\hline \# COS I0. D. Pesadillas (n/\%) & 140 & 43.7 & 15 & 4.9 & 0.001 \\
\hline Escala de somnolencia de Epworth $>10$ & 64 & 20 & 73 & 24 & 0.006 \\
\hline Cuestionario de SPI (n/\%) & 73 & 22.8 & 96 & 31.6 & 0.014 \\
\hline
\end{tabular}

al., 1996; Schussler et al., 2008), la edad mayor de 30 años (Facco et al., 2010; Hedman et al., 2002), la nuliparidad (Dorheim et al., 2014), el tabaquismo (Fernández-Alonso et al., 2012), la queja por tener que ocuparse de otros hijos durante la noche (Hutchison et al., 2012), como razones que justifican más insom- nio durante la gestación. Nosotros podemos afirmar que la edad y la queja por familiares, fueron motivos manifestados por nuestras ME, pero el insomnio lo comunicaron más las MNE que las ME.

En cuanto a otros trastornos del sueño, encontramos diferencia significativa entre el 1 er $\mathrm{T}$ y el 3 er $\mathrm{T}$ en 
lo relativo a la hipersomnia. Se sabe que este fenómeno se debe al aumento de secreción de progesterona en el 1er T. (Santiago et al., 2001; Lamberg, 2006) y por sueño fragmentado en el 2 do. T. Ahora bien, cuando se comparó el puntaje obtenido por ESS, el resultado fue significativo en las MNE, pero sin superar el punto de corte.

\section{Limitaciones del estudio}

Reconocemos que el presente estudio tiene limitaciones como, por ejemplo, las derivadas del uso de cuestionarios que pueden haber dado lugar a sesgos de información, sin embargo, es común su utilización en estudios epidemiológicos. Los datos obtenidos sobre el sueño pueden ser menos objetivos que constatar los mismos con polisomnografía, pero debido al tamaño de la muestra tal recurso hubiera sido impracticable. Sin embargo, la gran cantidad de ME participantes y sobre todo el compararlas con MNE, pueden considerarse como recursos válidos para compensar esa limitación.

\section{Conclusiones}

Las ME atribuyen su queja de dormir mal, a la presencia de sueño fragmentado por la mayoría de las causas indagadas. Los disturbios del sueño tales como los trastornos respiratorios durante el sueño, las pesadillas y los movimientos de piernas, fueron prevalentes en ME. La insatisfacción con el sueño y el insomnio fueron prevalentes en el 3er T. y la hipersomnia en el 1er T. Otros trastornos del sueño, tales como ciertos trastornos del movimiento (principalmente SPI y bruxismo), el insomnio y la hipersomnia, así como la satisfacción con el sueño, fueron más significativos en MNE.

Es importante que todos los miembros del equipo de salud, conozcan las características normales y los trastornos del sueño durante el embarazo para ofrecer una serie de pautas o recomendaciones que contribuyan a mejorar la salud física y mental presente y futura, de la gestante y del feto.

Conflictos de intereses: los autores declara no tener conflictos de intereses.

Agradecimientos: por la recolección de encuestas a la Secretaria Sra. Romina Ferraro.

Por la corrección del texto y aportes a los Dres. Carlos Schenck, Arturo Garay y Daniel Cardinali.

\section{Referencias bibliográficas}

Ali, R. A., Egan, L. J. (2007). Gastroesophageal reflux disease in pregnancy. Best Pract Res Clin Gastroenterol, 21(5):793-806. doi: 10.1016/j. bpg.2007.05.006.

Allen, R. P., Picchietti, D., Hening, W. A., Trenkwalder, C., Walters, A. S., Monplaisi J. (2003). Restless legs syndrome: diagnostic criteria, special considera-tions, and epidemiology. A report from the restless legs syndrome diagnosis and epidemiology workshop at the National Institutes of Health. Sleep Med, 4(2):101-19. doi: 10.1016/s1389-9457(03)00010-8.

Alvarez-Aguilar, D., Valero-Roncero, J., Pérez-Rodríguez, E., Sánchez-Márquez, G. (2010). Trastornos del sueño durante el embarazo. Matronas Prof, 11(1), 11-17.

Baratte-Beebe, K.R., Lee, K. (1999). Sources of midsleep awakenings in childbeaing women. Clin Nurs Res, 8(4):386-97. doi: 10.1177/10547739922158377. Bobes, J., González, M. P., Saiz, P. A., Bascarán, M.T., Iglesias, E., Fernández, J. M. (2000). Propiedades psicométricas del cuestionario Oviedo del sueño. Psicothema, 12(1), 107-12.

Calaora-Tournadre, D., Ragot, S., Meurice, J. C., Pourrat, O., D’Halluin, G., Magnin, G., Pierre, E. (2006). Obstructive sleep apnea syndrome during pregnancy: prevalence of main symptoms and relationship with pregnancy induced-hypertension and intra-uterine growth retardation. Rev Med Interne, 27(4), 291-5. doi: 10.1016/j.revmed.2006.01.015.

Domingo, C., Latorre, E., Mirapeix, R. M., Abad, J. (2006). Snoring, obstructive sleep apnea syndrome, and pregnancy. Int J Gynaecol Obstet, 93(1):57-9. doi: 10.1016/j.ijgo.2006.01.004.

Dorheim, S. K., Bjorvatn, B., Eberhard-Gran, M. (2014). Can insomnia in pregnancy predict postpartum depression? A longitudinal, population-based study. PLoS ONE. 9 (4):e94674. https://doi.org/10.1371/journal.pone.0094674

Dzaja, A., Arber, S., Hislop, J., Kerkhofs, M., Kopp, C., Pollmächer, T., Päivi Polo-Kantola, P., Skene, D. J., Stenuit, P., Tobler, I., Porkka-Heiskanen, T. (2005). Women's sleep in health and disease. J Psychiatr Res, 39(1), 55-76. doi: 10.1016/j.jpsychires.2004.05.008.

Ellis, J. G., Gehrman, P., Espie, C. I. E., Riemann, D., Perlis, M. L. (2012). Acute insomnia: current conceptualizations and future directions. Sleep Med Rev, 16(1), 5-14. doi: 10.1016/j.smrv.2011.02.002.

Facco, F.L., Kramer, J., Ho, K. H., Zee, P. C., Grobman, W. A. (2010). Sleep disturbances in pregnancy. Obstet Gynecol, 115(1):77-83. doi: 10.1097/ AOG.0b013e3181c4f8ec.

Facco, F., Louis, J., Knavert, M., Balserak, B. (2017). Sleep-disordered Breahting in Pregnancy. En M. Kryger T. Roth W. Dement (Eds.). Principles and Practice of Sleep Medicine. 6th edition. Elsevier.

Fernandez-Alonso, A. M., Trabalon-Pastor, M., Chedraui, P., Perez-López, F. (2012). Factors related to insomnia and sleepiness in the late third trimester of pregnancy. Arch Gynecol Obstet. 286(1), 55-61. doi: 10.1007/ s00404-012-2248-Z.

García, M. A., González, F., De León, J., Herráiz C. (2006). Prevalencia del síndrome de piernas inquietas en embarazadas. X Jornada Científica de Residentes del Hospital Virgen de la Luz, SESCAM. Cuenca: 28-30.

García-Portilla, M., Sáiz, P., Diaz-Mesa, E. et al. (2009). Rendimiento psicométrico del Cuestionario Oviedo en pacientes con trastorno mental grave. Rev Psiquiatr Salud Ment, 2(4), 169-177.

Guilleminault, C., Querra-Salva, M., Chowdhuri, S., Poyares, D. (2000). Normal pregnancy, daytime sleeping, snoring and blood pressure. Sleep Med, 1(4), 289-297. doi: 10.1016/s1389-9457(00)00046-0.

Hall, W.A., Hauck, Y.L., Carty, E.M., Hutton E. K., Fenwick, J., Stoll, K. (2009). Childbirth fear, anxiety, fatigue, and sleep deprivation in pregnant women. J Obstet Gynecol Neonatal Nurs, 38(5), 567-576. doi: 10.1111/j.15526909.2009.01054.x.

Hedman, C., Pohjasvaara, T., Tolonen, U., et al. (2002). Parasomnias decline during pregnancy. Acta Neurol Scan, 105(3), 209-214. doi: 10.1034/j.16000404.2002.10060.x. 
Hedman, C., Pohjasvaara, T., Tolonen, U., Suhonen-Malm, A. S., Myllylä, V. V. (2002). Effects of pregnancy on mothers'sleep. Sleep Med, 3(1), 37-42. doi: 10.1016/s1389-9457(01)00130-7.

Hening, W., Walters, A., Allen, R. P., Montplaisir, J., Myers, A., Ferini-Strambi, L. (2004). Impact, diagnosis and treatment of restless legs syndrome (RLS) in a primary care population: the REST (RLS epidemiology, symptoms, and treatment) primary care study. Sleep Med, 5(3):237-46. doi: 10.1016/j.sleep.2004.03.006.

Hutchison, B., Stone, P., McCowan, L. M., McCowan, L. M. E., Stewart, A. W., John MD Thompson, J. M. D., \& Mitchell, E. A. (2012). A postal survey of maternal sleep in late pregnancy. BMC Pregnancy Childbirth, 12:144. https://doi.org/10.1186/1471-2393-12-144

Johns, M. W. (1991). A new method for measuring daytime sleepiness the Epworth sleepiness scale. Sleep, 14(6):540-5. doi: 10.1093/sleep/14.6.540.

Kizilirmak, A., Timur, S., Kartal, B. (2012). Insomnia in pregnancy and factors related to insomnia. Scientific World Journal, 2012:197093. doi: 10.1100/2012/197093.

Köken, G., Kir, F., Cosar, E., Saylan, F., Yilmaz, N., Altuntas, I., Fidan, F., Unlu, M., Yilmazer, M. (2007). Oxidative stress markers in pregnant women who snore and fetal outcome: a case control study. Acta Obstet Gynecol Scand, 86(11), 1317-21. doi: 10.1080/00016340701662183.

Lamberg, L. (2006). Sleeping poorly while pregnant may not be "normal". JAMA, 295(12), 1357-1361. doi:10.1001/jama.295.12.1357.

Lancel, M., Faulhaber, J., Holsboer, F., Rupprecht, R. (1996). Progesterone induces changes in sleep comparable to those of agonistic GABAA receptor modulators. Am J Physiol, 271(4 Pt 1):E763-72. doi: 10.1152/ajpendo.1996.271.4.E763.

Langner, S., Halank, M., Kolditz, M., Schiemanck, S., Hoffken, G. (2007). Twin prenancy and severe obstructive sleep apnea. Geburtshilfe Neonatol, 211(2):93-7. doi: 10.1055/s-2006-942180.

Lee, K.A., Zaffke, M.E. (1999). Longitudinal changes in fatigue and energy during pregnancy and the postpartum period. J Obstet Gynecol Neonatal Nurs, 28(2):183-191. doi: 10.1111/j.1552-6909.1999.tb01983.x.

Lee, K.A., Gay, C.L. (2004). Sleep in late pregnancy predicts length of labor and type of delivery. Am J Obstet Gynecol, 191(6), 2041-6. doi:10.1016/j. ajog.2004.05.086.

Leigh Signal, T., Paine, S-J., Sweeney, B., Priston, M., Muller, D., Smith, A., Lee, K. A., Huthwaite, M., Reid, P., Gander, P. (2014). Prevalence of abnormal sleep duration and excessive daytime sleepiness in pregnancy and the role of socio-demographic factors: comparing pregnant women with women in the general population. Sleep Medicine, 15(12):1477-83. doi: 10.1016/j.sleep.2014.07.007.

Lopes, E.A., Carvalho, L.B., Seguro, P.B., Mattar, R., Silva, A.B., Prado, L.B., Fernandes do Prado, G. (2004). Sleep disorders in pregnancy. Arq Neuro-Psiquiatr, 62(2A), 217-21. doi: 10.1590/s0004-282x2004000200005. Lyn, J. (2007). Restless legs syndrome: pathophysiology and the role of iron and folate. Altern Med Rev, 12(2), 101-112. PMID: 17604457.

Malfertheiner, S. F., Malfertheiner, M.V., Kropf, S, Kropf, S., Costa, S-D., Malfertheiner, P. (2012). A prospective longitudinal cohort study: evolution of GERD symptoms during the course of pregnancy. BMC Gastroenterol. 12:131. doi: 10.1186/1471-230X-12-131.

Manber, R., Armitage, R. (1999). Sex, steroids, and sleep: a review. Sleep. 22(5):540-55. doi:10.1093/SLEEP/22.5.540.

Manconi, M., Govoni, V., Economou, N. T., Cesnik, E., Casetta, I., Mollica, G., Ferini-Strambi,L., Granieri, E. (2004). Restless legs syndrome and pregnancy. Neurology, 63(6), 1065-9. doi:10.1212/01.wnl.0000138427.83574.a6.

Meijer, A.M., Van den Wittenboer, G.L. (2007). Contribution to infants'sleep and crying to marital relationship of first-time parent copules in the1st. year after childbirth. J Fam Psychol, 21(1), 49-57. doi: 10.1037/0893$\underline{3200.21 .1 .49 .}$
Mindell, J.A., Jacobson, B.J. (2000). Sleep disturbances during pregnancy. J Obstet Gynecol Neonatal Nurs, 29(6), 590-597. doi: 10.1111/j.15526909.2000.tb02072.x.

Morey, A. K., Pedram, A., Razandi, M., Prins, B. A., Hu, R. M., Biesiada, E., Levin, E. R. (1997). Estrogen and progesterone inhibit vascular smooth muscle proliferation. Endocrinology, 138(8):3330-9. doi: 10.1210/ endo.138.8.5354.

National Sleep Foundation. (2007). (s/d). Sleep in America. http://www. sleepfoundation.org/sites/default/files/Summary_Of_Findings\%20-\%20 Último acceso: 3 de abril de 2021.

Neau, J.P., Texier, B., Ingrand, P. (2009). Sleep and vigilance disorders in pregnancy. Eur Neurol, 62(1), 23-29. doi: 10.1159/000215877.

Okun, M.L., Schetter, C.D., Glynn, L.M. (2011). Poor sleep quality is associated with preterm birth. Sleep, 34(11), 1493-1498. doi: 10.5665/ sleep.1384.

Pérez-Chada, D., Videla, A. J., O’Flaherty, M.E., Majul, C., Catalini, A. M., Caballer, C. A., Franklin K. A. (2007). Snoring, witnessed sleep apneas and pregnancy induced hypertension. Acta Obstet Gynecol Scand, 86(7):78892. doi: 10.1080/00016340701281919.

Persi, G. A., Etcheverry, J., Vecchi, C., Parisi, V., Ayarza, A., Gatto, E. (2009). Prevalence of restless legs syndrome: A community-based study from Argentina. Parkinsonism Relat Disord, 15(6):461-5. doi: 10.1016/j. parkreldis.2008.11.012.

Pien, G.W., Fife, D., Pack, A., Nkwuo, J. E., Schwab, R. J. (2005). Changes in symptoms of sleep-disordered breathing during pregnancy. Sleep, 28(10):1299-305. doi: 10.1093/sleep/28.10.1299.

Rey, E., Rodriguez-Artalejo, F., Herraiz, M. A., Sanchez, P., Alvarez-Sanchez, A., Escudero, M., Diaz-Rubio, M. (2007). Gastroesophageal reflux symptoms during and after pregnancy: a longitudinal study. Am J Gastroenterol, 102(11):2395-400. doi: 10.1111/j.1572-0241.2007.01452.x.

Roush, S. F., Bell, L. (2003). Obstructive sleep apnea in pregnancy. J Am Board Fam Pract, 17(4):292-4. doi: 10.3122/jabfm.17.4.292.

Santiago, J.R., Nolledo, M.S., Kinzler, W., Santiago, T.V. (2001). Sleep and sleep disorders in pregnancy. Ann Intern Med, 134(5), 396-408. doi: 10.7326/0003-4819-134-5-200103060-00012.

Schussler, P., Kluge, M., Yassouridis, A., et al. (2008). Progesterone reduces wakefulness in sleep EEG and has no effect on cognition in healthy postmenopausal women. Psychoneuroendocrinology, 33(8), 1124-1131. doi: 10.1016/j.psyneuen.2008.05.013.

The American College of Obstetricians and Gynecologists Committee Opinion no. 630. (2015) Screening for perinatal depression. Obstet Gynecol, 125(5), 1268-71. doi: 10.1097/01.AOG.0000465192.34779.dc.

Tsai, S.Y., Lin, J.W., Kuo, L.T., Thomas, K.A. (2012). Daily sleep and fatigue characteristics in nulliparous women during the third trimester of pregnancy. Sleep, 35(2), 257-262. doi: 10.5665/sleep.1634.

Tunc, T., Karadag, Y. S., Dogulu, F., Inan, L. E. (2007). Predisposing factors of Restless Legs Syndrome in pregnancy. Mov Disord, 22(5), 627-31. doi: $10.1002 / \mathrm{mds} .21291$.

Vgontzas, A. N., Bixler, E. O., Lin, H.M., Prolo, P., Mastorakos, G., Vela-Bueno, A., Kales, A., Chrousos, G. P. (2001). Chronic insomnia is associated with nyctohemeral activation of the hypothalamic-pituitary-adrenal axis: clinical implications. J Clin Endocrinol Metab, 86(8):3787-94. doi: 10.1210/ jcem.86.8.7778.

Watson, N.F., Goldberg, J., Arguelles, L., Buchwald, D. (2006). Genetic and environmental influences on insomnia, daytime sleepiness, and obesity in twins. Sleep, 29(5), 645-9. doi: 10.1093/sleep/29.5.645. 